\title{
Revisão Sistemática do Estudo da Dinâmica de Estados Excitados da Tetraazaporfirina de Cobalto usando Surface Hopping
}

\author{
Igor C. Morais, João L. P. Lisboa, José A. S. Neto, Karolina S. \\ Gonçalves, Oliver A. J. S. Filho \& Rosemberg F. N. Rodrigues
}

A terapia fotodinâmica (TFD) é uma reação fotoquímica que atua diretamente em tecidos tumorais com intuito de destruí-los, quando aplicada de maneira ideal, sem afetar o tecido saudável. Caso um sensibilizador localize um tecido tumoral por este é anulado, devido à produção de espécies tóxicas como resultado da transferência de energia da molécula para o tecido tumoral, destruindo a anomalia de forma segura. Em sistemas naturais, a biossíntese do óxido de nitrogênio (NO) e suas várias reações biológicas e fisiológicas ocorrem através da interação com metaloproteínas de ferro, rutênio, cobre, cobalto, dentre outros metais de transição. Assim, as reações de NO através de sua coordenação com centros de metal de transição possuem grande potencial e são principais candidatos para cumprir essa função, podem ser citados porfirinas e seus derivados como a tetraazaporfirina.

Palavras-chave: tetrazaporfirina; surface hopping; estados excitados.

Photodynamic therapy (PDT) is a photochemical reaction that acts directly on tumor tissues in order to destroy them, when applied in ideal ways, without affecting healthy tissue. If a sensitizer finds a tumor tissue, it is canceled, due to the production of toxic species as a result of the energy transfer of the molecule to the tumor tissue, safely destroying the anomaly. In natural systems, nitrogen oxide NO biosynthesis and its various biological and physiological reactions occur through the interaction with iron, ruthenium, copper, cobalt metalloproteins, among other transition metals. Thus, the reactions of NO through its coordination with transition metal centers have great potential and are the main candidates to fulfill this function, being able to mention porphyrins and their derivatives such as tetraazaporphyrin.

Keywords: tetraazoporphyrin; surface hopping; excited states. 


\section{Introdução}

A terapia fotodinâmica (TFD) atua diretamente em tecidos tumorais com intuito de destruí-los, quando aplicada de maneira ideal, mas sem prejudicar o tecido saudável, deixando-o natural, sem danificá-lo. Dessa forma, o fotossensibilizador não causa danos em tecidos vigorosos, pois não tem efeito tóxico ou anormal na falta de luz. Caso um sensibilizador localize um tecido tumoral por este é anulado, devido à produção de espécies tóxicas com resultado da transferência de energia, assim, o tecido é destruído ${ }^{1}$.

Visando a aplicações na área de fotodinâmica, a estrutura do óxido de nitrogênio (NO) também chamado de óxido nítrico ou monóxido de nitrogênio, é bastante conhecida por desempenhar papéis diversos em processos biológicos descrita na literatura, como reguladores primários do tônus vascular, está relacionada na transdução de sinal no sistema nervoso central e periférico. A estrutura do óxido de nitrogênio (NO) é ainda parte integrante do sistema de resposta imunológica associada à ativação de macrófagos e neutrófilos, dentre outros processos ${ }^{2,3}$.

Em sistemas naturais, a biossíntese de NO e suas várias reações biológicas e fisiológicas ocorrem através da interação com metaloproteínas de ferro, rutênio, cobre, cobalto, dentre outros metais de transição. Assim, as reações de NO, através de sua coordenação com centros de metal de transição que possuem grande potencial e são principais candidatos para cumprir essa função, podendo citar porfirinas e seus derivados como a tetraazaporfirina $a^{4,5,6}$.

A característica fundamental das porfirinas é a sua formação estrutural constituída por quatro anéis pirrólicos ligados por carbonos, cuja disposição é altamente conjugada. Esse trabalho visa ao estudo de um de seus derivados, a tetraazaporfirina, conhecida também como TAPs, Figura 1 (a), no qual substituem-se quatro nitrogênios nas posições meso. Existem outros derivados como as fitalocianinas, no qual são acrescentados anéis benzênicos ligados aos anéis pirrólicos da tetraazaporfirinas, entre outros derivados devido à inclusão de ligantes na estrutura base da porfirina, conhecidos como metaloporfirinas, podendo ser citada a inclusão de um metal no sítio central como ferro, rutênio, cobre, cobalto, mostrado na Figura 1 (b), dentre outros. Sensores químicos ou até possíveis tratamentos contra o câncer ou HIV apresentam grande potencial tecnológico e farmacológico dessas estruturas e o real interesse do meio científico em estudá-las, levando ao aumento de pesquisas desenvolvidas $^{7,8}$.

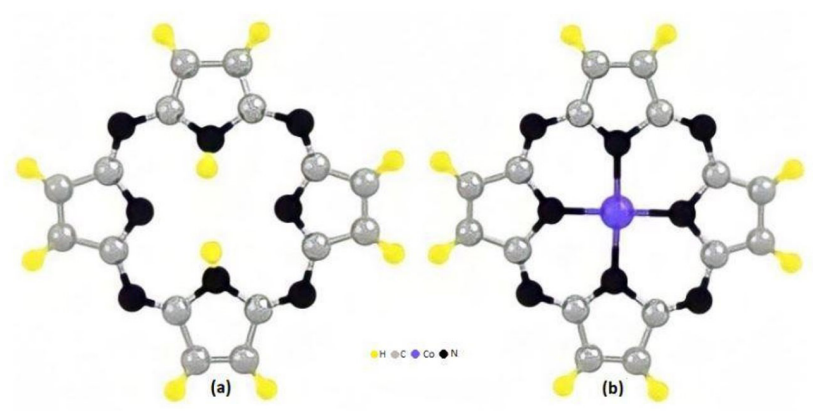

Figura 1: (a) - Estrutura da Tetraazaporfirina; (b) - Estrutura da Tetraazaporfirina de Cobalto. Fonte: Autores.

Tetraazaporfirinas possuem características que despertam interesse em aplicação como sensitizadores em terapia fotodinâmica, através da interação com a radiação com comprimento de onda e frequência bem definidos com intuito de promover a morte do tecido tumoral. Essas particularidades, como baixa toxicidade, estabilidade térmica, facilidade de síntese, podem atuar como transportadores de NO como mostra a Figura 2 (a), de tal forma a atuar em regiões específicas e agindo conforme exposição à radiação eletromagnética, pois espécies tóxicas geradas com resultado da transferência de energia podem resultar em tecidos tumorais destruídos. Outra peculiaridade das TAPs é a possibilidade de alterar o potencial de redução devido à troca de substituinte, assim controla-se a labilidade da ligação Metal-NO, em certo estado de oxidação, dependendo do ligante interagindo com o metal. Dessa forma é possível aplicar algumas variações para o ligante $\mathrm{R}$ na Tetraazaporfirina de Cobalto 
(Figura 2 (b)) ou realizar a variação do estado de oxidação. Lima e colaboradores desenvolveram um trabalho sobre a Tetraazaporfirina de Rutênio em 2013 e relataram que os parâmetros geométricos e eletrônicos da ligação Ru-NO são altamente dependentes do estado de oxidação do $\mathrm{Ru}$ e da natureza química do ligante $\mathrm{L}$ na posição axial. Os resultados também mostraram que RuTAP e Ru (L) TAP são bons candidatos potenciais para serem usados como portadores de $\mathrm{NO}$ em organismos vivos ${ }^{9}$.
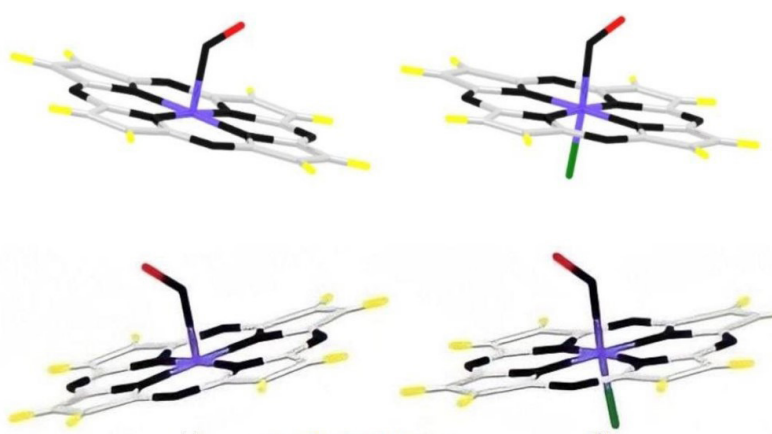

(a)

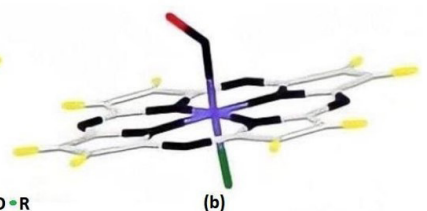

Figura 2: (a) Estrutura da Tetraazaporfirina de Cobalto com óxido nítrico. (b) Estrutura da Tetraazaporfirina de Cobalto com óxido nítrico e ligante a ser definido. Fonte: Autores.

Com aplicação da Tetraazaporfirina de Cobalto em fotodinâmica surge o questionamento de como essa estrutura se comporta perante a absorção dessa energia, emitida com frequência e comprimento de onda bem definidos em determinada região do organismo. Essa resposta pode surgir através da mecânica quântica proveniente de cálculos teóricos e computacionais (Teoria de Estados Excitados). Motivada pelos avanços algoritmos, a pesquisa computacional sobre simulações dinâmicas de sistemas moleculares eletronicamente excitados vem se desenvolvendo rapidamente nas últimas décadas, como mostra a Figura 3 (a), no qual a quantidade de publicações X ano evidencia o aumento considerável, quando comparado ao ano anterior. Sistemas maiores e mais complexos são exibidos diariamente em todo o mundo Figura 3 (b) e por grupos de pesquisa em diversas áreas, Figura 3 (c), proporcionando informações fundamentais para interpretar fenômenos de estados excitados. São revelados por técnicas espectroscópicas avançadas, para explicar processos fotoquímicos diversos.

Vários métodos estão disponíveis para simulações da dinâmica de estado excitado não adiabático, a partir da propagação completa das funções de ondas eletrônicas ${ }^{10}$, que requer predefinição de superfícies de energia potencial multidimensional, para aproximações semi-clássicas, que reduzem a propagação da função de onda para ensembles de trajetórias independentes baseadas apenas em propriedades locais ${ }^{11}$.

Do ponto de vista das simulações semi-clássicas não dinâmicas da dinâmica do estado excitado, o método ideal para cálculos de estrutura eletrônica deve satisfazer aos seguintes critérios: seja computacionalmente rápido; forneça energias para estados excitados de diferentes naturezas com precisão similar; forneça gradientes confiáveis (preferencialmente analíticos) para estados excitados; permita o cálculo de estruturas eletrônicas perto da interseção com o estado fundamental; permita o cálculo de estruturas eletrônicas no limite da interseção entre estados excitados; seja independente da intervenção humana para executar grandes conjuntos de diferentes geometrias.

Com precisões diferentes, os métodos para cálculo de estados excitados baseados em DFT cumprem a maioria desses critérios, especialmente a eficiência computacional. Contudo, esses métodos, geralmente, falham para o critério de descrição perto da interseção com o estado fundamental. No entanto, ainda considerando os prós e os contras, o Surface Hopping com base nas excitações de DFT (SH/DFT) é uma boa alternativa para simulações não adiabáticas, sob a condição de ser aplicada criticamente, tendo em mente todas essas restrições e limitações.

No Surface Hopping, a propagação do tempo do pacote de ondas quânticas é aproximada por um gama de trajetórias semi-clássicas que evoluem nas superfícies de Born-Oppenheimer de múltiplos estados eletrônicos. Eventos não adiabáticos (transferência de densidade de pacotes de ondas entre os estados) ${ }^{12}$, são simulados por um algoritmo estocástico que permite que cada trajetória salte para outros estados durante a propagação. Assim, espera- 
se que as estatísticas sobre o conjunto de trajetórias em termos de fração de trajetórias em cada estado eletrônico em cada etapa do tempo sejam uma representação aproximada da distribuição da densidade de pacotes de onda entre os estados excitados em função do tempo. $\mathrm{O}$ método foi proposto conceitualmente por Nikitin ${ }^{13}$ e teve suas primeiras implementações feitas por Tully e Preston ${ }^{14}$. Foi revisado recentemente por Curchod e colaboradores ${ }^{15} \mathrm{e}$ Akimov e colaboradores ${ }^{16}$. Portanto, definiu-se inicialmente o objetivo geral do trabalho para ser usado como solução da indagação apresentada e como base de análise dos artigos encontrados nas buscas.

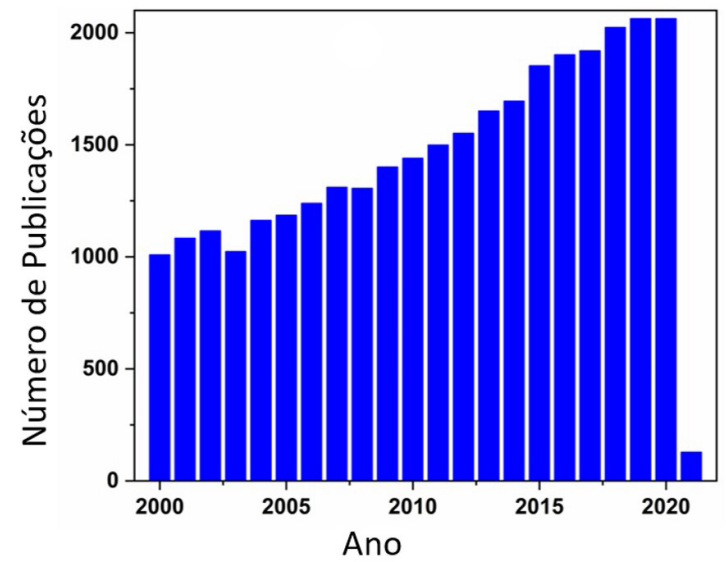

(a)

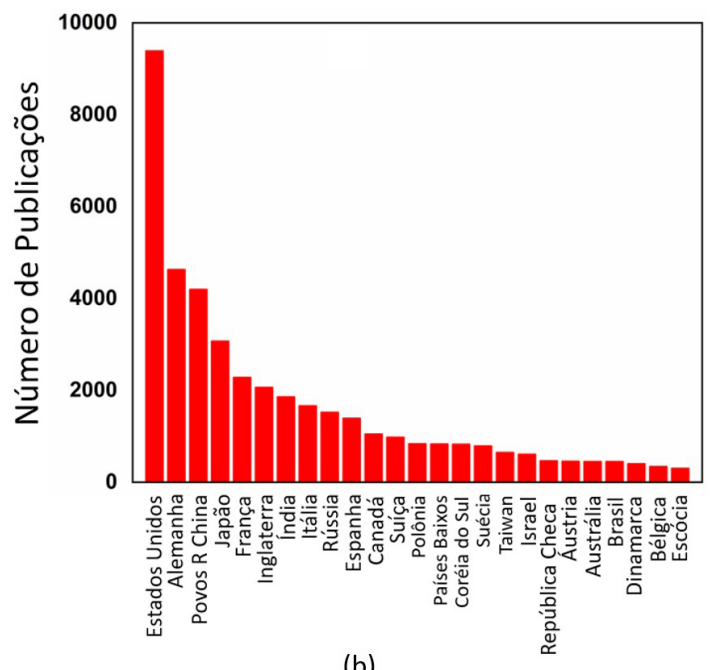

(b)

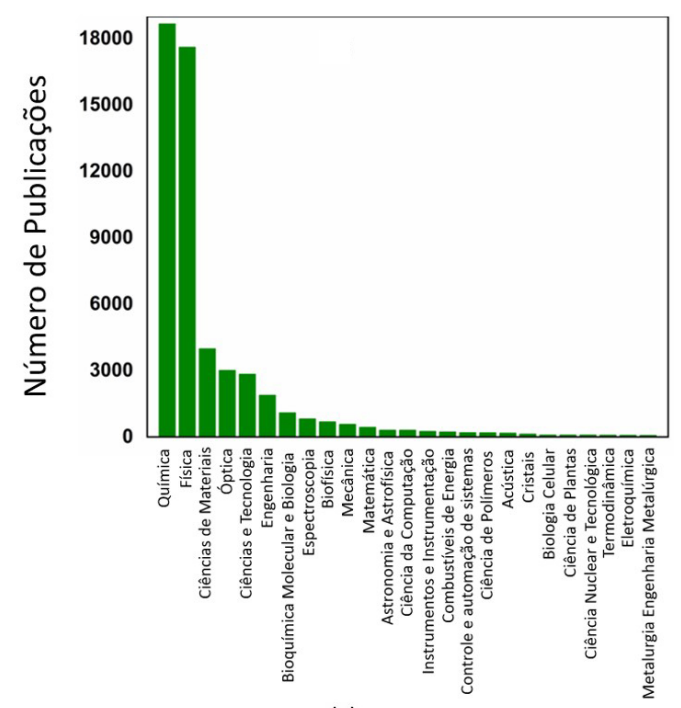

(c)

Figura 3: (a) Gráfico da evolução da produção científica sobre dinâmica de estados excitados no mundo nas últimas décadas. (b) Gráfico da produção científica por países sobre dinâmica de estados excitados no mundo nas últimas décadas. (c) Gráfico da produção científica por áreas de atuação sobre dinâmica de estados excitados no mundo nas últimas décadas. Fonte: Autores.

\section{Metodologia}

Para esta revisão bibliográfica sistemática, definiu-se inicialmente o objetivo geral do trabalho para ser usado como solução da indagação apresentada na introdução e como base de análise dos artigos encontrados nas buscas. Desta forma, as buscas por trabalhos que correlacionaram com o tema proposto por este artigo foram realizadas nas bases de dados Web of Science, Springer, Science Direct, PubMed e Google Academic, por apresentarem em suas estruturas de pesquisa mecanismos que destacam e filtram publicações relevantes ao tema. Essa busca teve o intuito de verificar as principais palavras-chave utilizadas pelos autores, assim este processo ajuda a aprimorar a pesquisa diminuindo a quantidade de trabalhos, mas concentrando os mais relevantes para o tema. Durante o processo, utilizaram-se formulações de termos e operadores, como: "cobalt; tetraazaporphyrin; excited state dynamics; surface hopping; photodynamic", "excited state dynamics AND photodynamic AND tetraazaporphyrin" e "surface hopping 
AND excited state dynamics AND tetraazaporphyrin AND photodynamic". Assim, definiu-se a string de busca, na qual funciona corretamente com o operador lógico AND, além de ser aplicável em todas as bases. Esta string se limitou a apresentar termos como, cobalto (cobalt), tetraazaporfirina (tetraazaporphyrin), salto de superfície (surface hopping), "dinâmica dos estados excitados" ("excited state dynamics") e fotodinâmica (photodynamic). Tendo em vista que para pesquisa por publicações, filtraram-se somente artigos científicos de língua inglesa, além de limitar o período de busca em 50 anos.

A Figura 4 apresenta a etapa de seleção dos artigos por meio do uso do programa StArt, sendo que este fluxograma foi desenvolvido com cunho educativo exibindo as principais seções da ferramenta.

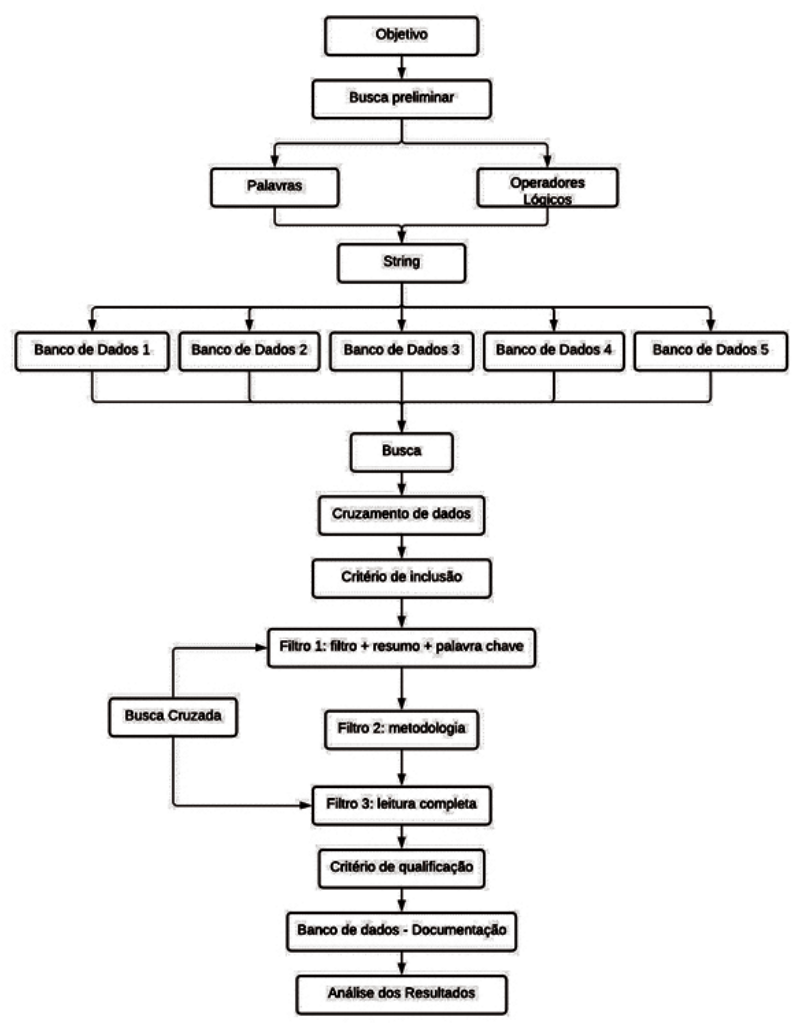

Figura 4: Fluxograma da metodologia. Fonte: Autores.
Abordagens mistas quânticas-clássicas são provavelmente a classe de métodos mais empregada para realizar simulações de dinâmica molecular em estado excitado, incluindo efeitos não-adiabáticos. Nessas abordagens, que incluem o salto de superfície e os métodos de Ehrenfest, a evolução do tempo nuclear é tratada classicamente, enquanto a evolução do tempo da população de cada estado eletrônico é tratada separadamente. No salto de superfície, a evolução temporal da população é obtida em duas etapas: primeiro, a probabilidade de transição não-adiabática entre cada par de estados é calculada e um algoritmo estocástico é aplicado para decidir em qual estado a trajetória clássica é propagada na próxima etapa de tempo. Em segundo lugar, as estatísticas sobre um grande conjunto de trajetórias computadas independentemente permitem obter a fração das trajetórias (ocupação) em cada estado em função do tempo. A principal hipótese subjacente à abordagem de salto de superfície é que a ocupação e a população quântica de cada estado eletrônico são as mesmas se um número infinito de trajetórias forem calculadas.

Após a busca, com a ajuda da ferramenta, realizou-se um cruzamento de dados de ambas bases com o propósito de verificar a possível duplicação de trabalhos, para então diminuir a quantidade de publicações analisadas durante a aplicação dos critérios de inclusão.

Nesse sentido, como critério de inclusão, buscouse filtrar as publicações por 3 filtros distintos, conforme apresentados no fluxograma da Figura 4. No primeiro filtro, executou-se a leitura do título, resumo e palavraschave, com o intuito de identificar semelhança entre o objetivo da revisão sistemática e o apresentado no artigo. No segundo, realizou-se a leitura da metodologia dos artigos selecionados, objetivando escolher produções científicas que demonstrassem uma parte experimental mais elaborada, dando ênfase aos trabalhos que abordassem análise de dados econômicos do processo. Por fim, no terceiro filtro, verificaram-se os artigos selecionados por meio de uma leitura completa com o propósito de analisar os resultados e conclusões, as quais se interligam à proposta da metodologia definida pelo autor. Além disso, durante esta etapa estudaram-se os critérios de qualificação como 
a quantidade de citações, qualidade do periódico, ano de publicação, variabilidade de amostragem do experimento e H-Index da autoria. Para a aplicação de ambos os critérios utilizou-se a ferramenta StArt, na qual facilita a separação dos artigos em cada fase, além de proporcionar uma melhor análise dos trabalhos finais desta revisão. Nesse sentido, esta ferramenta, conforme exibe a Figura 5, é dividida em
5 seções, o: planejamento, execução, seleção, extração e sumarização. O planejamento define a base estrutural da revisão sistemática, na qual são determinado as bases de dados e os critérios de inclusão.

Já a execução é a etapa de importação de dados de cada base, apresentando o resultado bruto. Por outro lado, a seleção e a extração representam a primeira e última etapa de

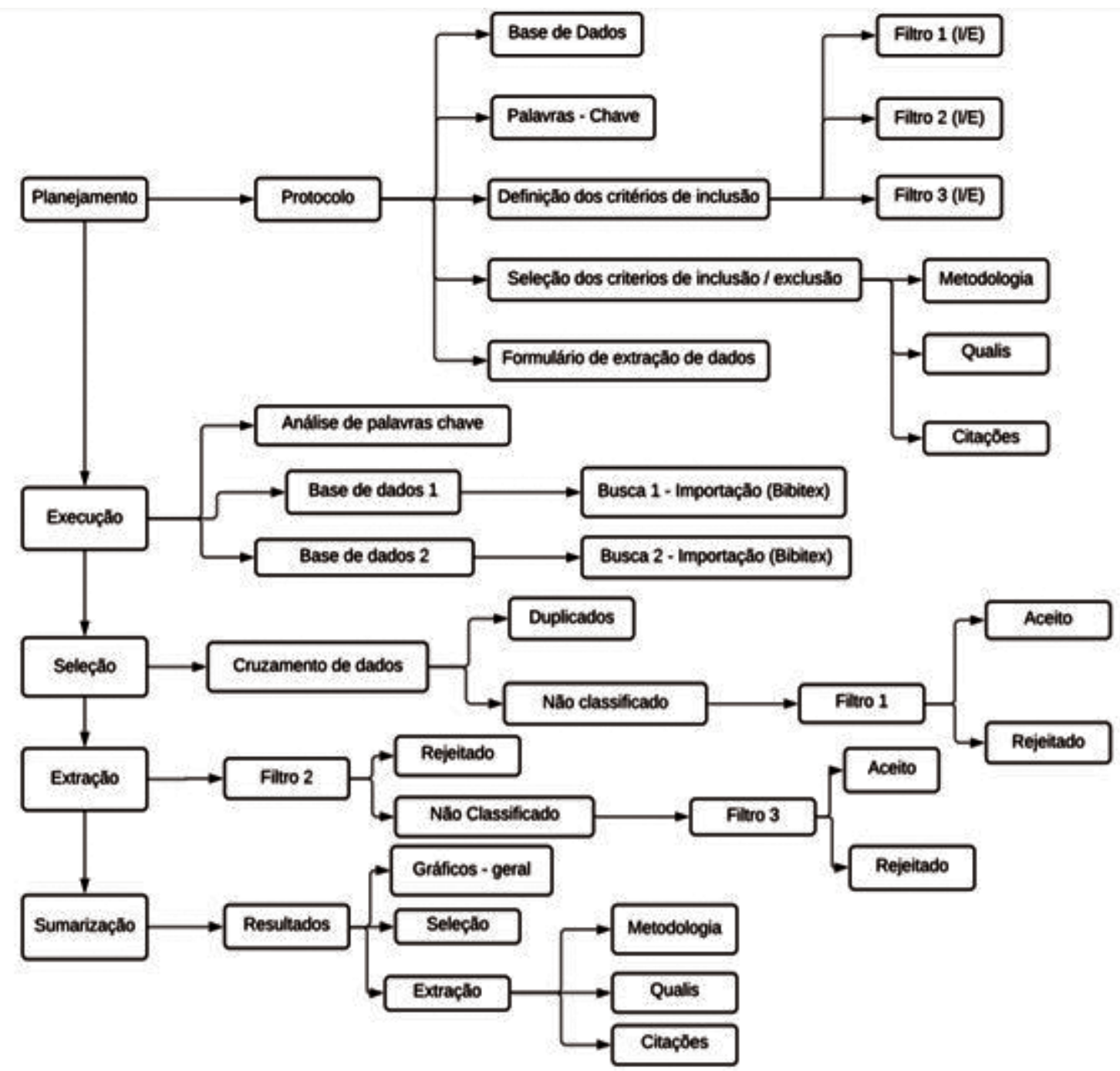

Figura 5: Fluxograma do procedimento. Fonte: Autores. 
aplicação dos critérios de inclusão, respectivamente. Por fim, a sumarização exibe o resumo gráfico em formato de mapas mentais, dos resultados da seleção dos artigos de cada etapa.

Assim, ao final do processo de inclusão, realizou-se nos artigos selecionados uma busca cruzada, na qual objetivouse identificar no referencial bibliográfico, de cada trabalho, possíveis materiais que passaram despercebidos pelos procedimentos de busca. Nesse sentido, analisou-se cada referência de acordo com os filtros determinados nesta metodologia.

Logo, foi elaborado um modelo ilustrado na Tabela 1 com o objetivo de identificar e limitar o número de artigos excluídos e a taxa de aproveitamento de cada artigo após o cruzamento de dados. Com o auxílio do Mendeley, foi registrado o material aprovado no primeiro filtro, otimizando assim a leitura das publicações necessárias para os próximos filtros, dito isso, para cada filtro eram retirados os artigos rejeitados. Finalizado o processo de busca, análise e documentação, desenvolveu-se uma síntese dos resultados obtidos do processo de seleção, expondo os dados da seguinte forma: quantidade de publicações por ano de cada base, artigos relevantes para o tema proposto deste trabalho, principais autores, países que mais publicaram sobre o tema, principais metodologias utilizadas, dando ênfase nos resultados das pesquisas científicas selecionadas, por fim realizando a análise econômica dos procedimentos utilizados pelos autores, buscando identificar se estes processos apresentam viabilidade econômica como um novo método da utilização dos estados excitados da tetraazaporfirina de cobalto, através do método surface.

\section{Resultados}

O projeto de pesquisa sofreu alterações devido à revisão sistemática realizada no início do trabalho. Inicialmente, o objetivo era a investigação do papel do taquisterol na regulação fotofísica/fotoquímica da fotossíntese da vitamina $\mathrm{D}$, através de dinâmica de estados excitados abordando suas propriedades de absorção eletrônica com abordagem via DFT e possivelmente outros níveis de teoria. Cecília Cisneros e colaboradores publicaram em $2017^{17} \mathrm{um}$ artigo cujo objetivo era investigar a influência do taquisterol na produção de vitamina $\mathrm{D}$ fotoquímica, visto que poucas pesquisas foram feitas para avaliar essa estrutura. Em particular, sua dinâmica de estado excitado nunca havia sido investigada por experimentos ou simulações. $\mathrm{O}$ taquisterol é formado principalmente pela excitação de ultravioleta de pré vitamina $\mathrm{D}$.

Os cálculos deste trabalho foram realizados com o pacote de química quântica da TURBOMOLE. O estado de menor energia, o fundamental, foi calculado via teoria do funcional da densidade (DFT), no qual foram executados usando o módulo dscf. Para níveis mais altos de energia, ou seja, estado excitado, foi empregado a implementação da Teoria do Funcional da Densidade Dependente do Tempo (TDDFT). Os cálculos da dinâmica molecular foram realizados usando o algoritmo Verlet e a dinâmica não adiabática foi realizada usando o método de SurfaceHopping (TDDFT-SH).

Neste trabalho, foram analisadas 87907 estruturas do conjunto de $300 \mathrm{~K}$ obtidas a partir da simulação de REMD (Replica Exchange Molecular Dynamics) e classificadas

Tabela 1: Formulário para banco de dados. Fonte: Autores.

\begin{tabular}{|c|c|c|c|c|c|}
\hline Filtro & & $\begin{array}{l}\text { Quantidade de artigos } \\
\text { selecionados }\end{array}$ & & \multicolumn{2}{|c|}{$\begin{array}{l}\text { Quantidade de artigos } \\
\text { excluídos }\end{array}$} \\
\hline Data de busca & & $\begin{array}{l}\text { Quantidade de artigos } \\
\text { selecionados }\end{array}$ & & \multicolumn{2}{|c|}{ Taxa de aproveitamento } \\
\hline & & Encontrados & Filtro 1 & Filtro 2 & Filtro 3 \\
\hline & Ano & Quantidade de artigos & Selecionados & Selecionados & Selecionados \\
\hline
\end{tabular}


de acordo com a sua conformação em ângulo diedro em quatro diferentes rotâmeros: $\mathrm{tEt}, \mathrm{cEt}$, tEc e cEc (Figura 6). Os rotâmeros tEc estão presentes com o maior peso estatístico de $46 \%$, seguido pelos rotâmeros tEt (26\%). Os rotâmeros cEc e cEt representam $15 \%$ e $13 \%$ das estruturas, respectivamente.

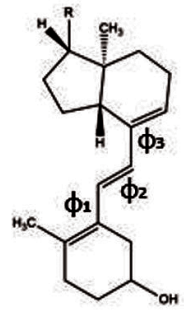

tEt

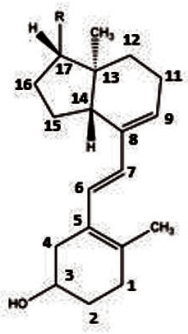

cEt

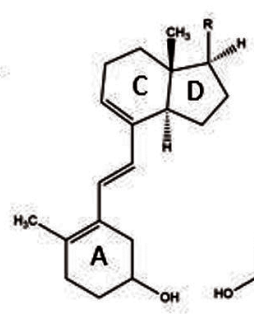

tEC

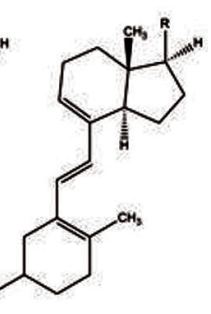

CEC
Figura 6: Rotâmeros de taquisterol. Fonte: The role of tachysterol in vitamin D photosynthesis-a non-adiabatic molecular dynamics study.

Cecília ainda afirma em caráter conclusivo que devido ao seu grande coeficiente de extinção e comportamento predominantemente não-reativo, o taquisterol age principalmente como um escudo solar suprimindo a formação de pré vitamina $\mathrm{D}$ de Pro e Lumi, uma vez que absorve na mesma região espectral. $\mathrm{O}$ taquisterol apresenta uma formação mais forte de toxisterol do que a pré vitamina $\mathrm{D}$ e pode ser visto como a principal rota de degradação da vitamina D. Apesar de ser pouco reativo, uma pequena quantidade de taquisterol reage à pré vitamina $\mathrm{D}$, que é favorecida pelos rotâmeros cEc no lado vermelho do pico de absorção de taquicardia máximo e pode, portanto, constituir um reservatório de pré vitamina $\mathrm{D}$ para os tempos em que apenas a radiação UV de baixa energia está presente.

Esses resultados mostram que a pesquisa que seria desenvolvida já estava publicada, dessa forma foi necessário a alteração do tema e isso ocorreu devido a revisão sistemática inicial. Dessa maneira iniciou-se uma nova revisão no qual foram encontrados 52 trabalhos utilizando as strings descritas na seção anterior. No qual 2 artigos estavam duplicados devido a busca em bases diferentes, 41 artigos foram rejeitados após a leitura de título e resumo por terem focos diferentes ou abordagem distintas. Após esse refinamento, restaram 9 artigos selecionados para leitura completa por estarem dentro do viés da pesquisa estabelecida. Finalizada a busca dos trabalhos ainda adicionou-se um artigo manualmente, pois este não foi relacionado a partir das strings selecionadas, mas de extrema importância devido à semelhança de compostos estudados, no qual refere-se a Tetraazaporfirina de Rutênio publicado por Lima e colaboradores em $2013^{9}$.

A importância da interação entre o óxido nítrico e Tetraazaporfirina de Rutênio despertou o interesse desses pesquisadores para realizarem um estudo teórico para os estados de oxidação $+1,+2$ e +3 de $R u$ para verificar se $\mathrm{Ru}$ (NO) TAP e $\mathrm{Ru}(\mathrm{NO})$ (L) TAP ( $\mathrm{LOCl}^{-}, \mathrm{NH} 3$ ou Py) podem ser usados como portadores de $\mathrm{NO}$ em sistemas biológicos. O trabalho foi realizado aplicando a teoria do funcional de densidade de Kohn-Sham (DFT) com troca híbrida de três parâmetros de Beck, funcional de correlação, conhecida como B3LYP e o conjunto de bases $6-31+\mathrm{G}(\mathrm{d}) /$ LANL2DZ. Os mínimos locais da hipersuperfície de energia potencial foram caracterizados pelo cálculo das frequências harmônicas vibracionais no mesmo nível de teoria usado nas otimizações de geometria com as representações de todas imagens trabalhadas na Figura 7.

Os resultados do cálculo realizado nos complexos RuTAP, [(NO) RuTAP], [(NO) Ru (L) TAP] ( $\mathrm{LOCl}^{-}, \mathrm{NH} 3$, Py) para os estados de oxidação $+1,+2$ e +3 de Ru no nível B3LYP / 6-31 + G (d) / LANL2DZ mostram que a ligação Ru-NO é energeticamente estável e as energias de ligação são altamente dependentes do estado de oxidação de Ru e da natureza química do ligante L. Um fato interessante é que os parâmetros geométricos do ligante TAP não dependem dos estados de oxidação $+1,+2$ e +3 dos ligantes $\mathrm{Ru}$ e L estudados. Isso significa que o ligante TAP deve manter sua estabilidade química característica nos complexos. Estes resultados teóricos mostram que os complexos [(NO) RuTAP], [(NO) Ru (L) TAP] são bons candidatos para uso como portadores de óxido nítrico em organismos vivos.

Buddhadev Maiti e colaboradores ${ }^{18}$ publicaram em 2016 um artigo, no qual abordaram a clivagem fotoinduzida da ligação entre o átomo de Si central em macrociclos de porfirina e o átomo de carbono vizinho de um ligante de 


\section{Artigo Convidado 3}

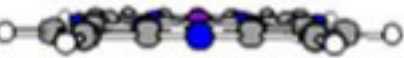

a)<smiles>[Te][Ba][Te]</smiles>

b)

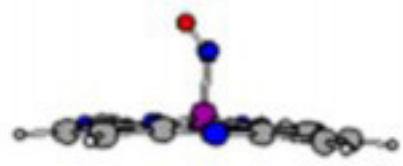

$\left[(\text { NO)RUTAP }]^{1-}\right.$

c)

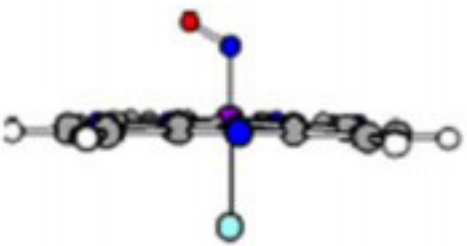

$[(\mathrm{NO})(\mathrm{Cl}) \mathrm{RUTAP}]^{2-}$

d)

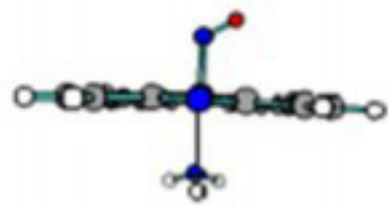

$\left[(\mathrm{NO})\left(\mathrm{NH}_{3}\right) \text { RuTAP] }\right]^{1}$

e)

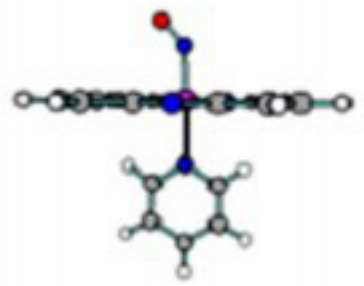

$\left[\left(N_{O}\right) \text { (PY)RuTAP }\right]^{1-}$

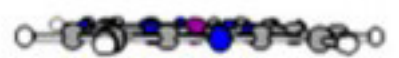

[RUTAP]

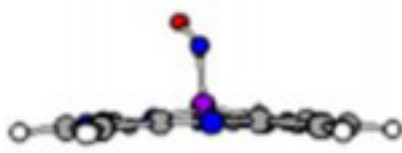

$[(N O) R U T A P]^{0}$

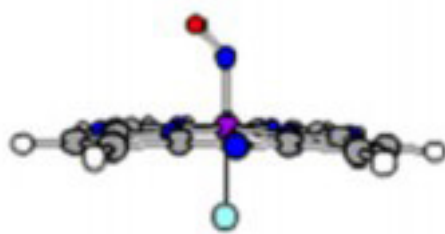

[(NO)(CI)RUTAP $]^{1-}$

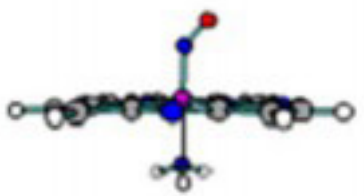

$\left[\left(\mathrm{NO}^{\mathrm{O}}\left(\mathrm{NH}_{3}\right) \mathrm{RUTAP}^{0}\right.\right.$

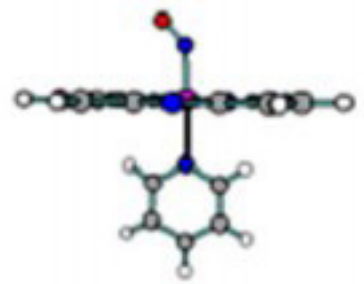

$\left[\left(\right.\right.$ NO) $(\text { Py)RUTAP }]^{0}$

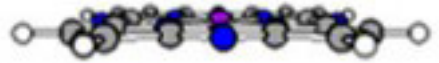

[RUTAP] $^{1+}$

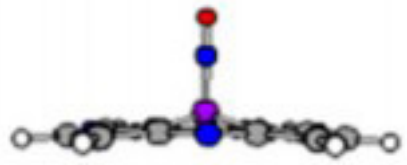

$[(N O) R U T A P]^{1+}$

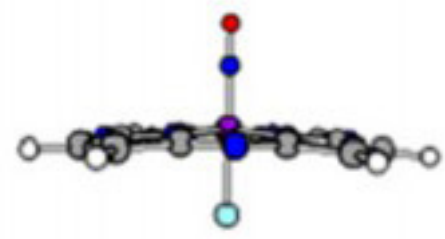

[(NO)(Cl)RUTAP] ${ }^{0}$

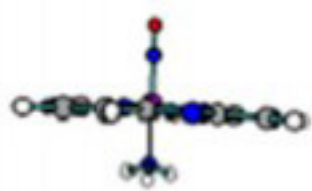

$\left[(\mathrm{NO})\left(\mathrm{NH}_{3}\right) \mathrm{RUTAP}^{1+}\right.$

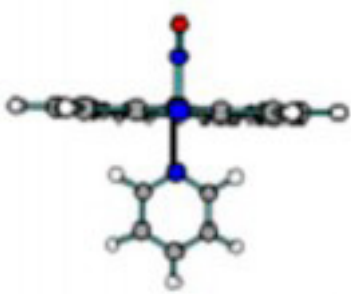

$\left[(\mathrm{NO})(\mathrm{Py}) \mathrm{RUTAP}^{1+}\right.$

Figura 7: Representação gráfica das geometrias moleculares totalmente otimizadas no nível B3LYP / 6-31 + G (d) / LANL2DZ para todos os complexos estudados. Fonte: Theoretical investigation on ruthenium tetraazaporphyrin as potential nitric oxide carrier in biological systems. 
alquilo axial é investigada por ferramentas experimentais e computacionais. Buddhadev utilizou cálculos de estrutura eletrônica, fornecendo informações sobre a natureza do estado excitado e o mecanismo de fotoclivagem resultante.

O trabalho publicado em 2005 por Danilo e colaboradores $^{19}$ relatou as propriedades limitadoras ópticas dos complexos axialmente substituídos de tetrapyrazinotetraazaporphyrinato (Pyz4TAP) com óxido de titânio, óxido de vanádio e dihidróxido de zircônio. A otimização das macrociclo abordadas ocorreu via teoria do funcional de densidade, utilizando o funcional de correlação de troca B3PW91. Cálculos de Kohn-Sham restritos e irrestritos foram realizados com o pacote Gaussian 98 para sistemas de shell aberto e fechado, respectivamente. $\mathrm{O}$ conjunto de bases $6-31 \mathrm{G}$ * foi consistentemente empregado para os complexos de vanadil e titanila alinhando desempenho e custo computacional. Para o átomo $\mathrm{Zr}$, Danilo utilizou conjunto de bases 3-21G para descrição do complexo Pyz4TAPZr $(\mathrm{OH})_{2}$ desse elemento. DFT também foi utilizado para porfirina livre de metais, devido à concordância encontrada entre os comprimentos das ligações experimentais e ângulos de ligação e os valores calculados correspondentes. Danilo e colaboradores efetuaram a substituição axial nos complexos metálicos tetravalentes, juntamente com a presença de nitrogênio nos anéis condensados do ligante macrocíclico, mostrando uma significativa melhora do efeito de limitação óptica (OL) na faixa visível quando comparada com as ftalocianinas análogas em similares condições experimentais. O grupo conclui que as variações do momento de dipolo e transições de estados excitados explicam essa melhoria significativa.

$\mathrm{O}$ artigo publicado por Pyosang e colaboradores ${ }^{20}$ trata de uma análise experimental e teórica de estruturas sintetizadas de benzoporfirinas, monobenzo- (Bp1), dibenzo- (syn Bp2 e antiBp2), tribenzo- (Bp3) e tetrabenzoporfirina (Bp4), sem substituintes periféricos. Os cálculos teóricos foram realizados através do programa Gaussian 03. DFT foi utilizado nesse trabalho para otimização das estruturas com os funcionais de troca híbrida de três parâmetros de Becke e a correlação funcional de Lee Yang-Parr (B3LYP), empregando conjunto de bases $6-31 G$ (d). O grupo investigou de forma qualitativa o efeito de anéis fundidos no estado eletrônico e na dinâmica de relaxamento de energia intramolecular, foram calculados os estados excitados usando TD-DFT no nível B3LYP / 6- 31G (d), a partir dos quais a energia de transição, força do oscilador e direção do momento de dipolo de transição puderam ser obtidas.

Zhihua e colaboradores ${ }^{21}$ publicaram um artigo em 2015 com objetivo de investigar os mecanismos de transferência de energia dos pontos quânticos de CdSe (QDs) aos derivados de porfirina com um potencial sistema de antenas com maior eficiência na coleta de luz. O grupo de pesquisadores abordaram os cálculos de transferência de energia de ressonância (FRET) e intensidade de fotoluminescência (PL). Esses tipos de cálculos indicam que maior transferência de energia pode ser alcançada com menor tamanho de pontos quânticos. Esse estudo sugere que os pontos quânticos de CdSe podem ser doadores promissores de transferência de energia para tetraazaporphyrins e absorvedores de NIR para formar sistemas de antenas com maior eficiência de coleta de luz.

A busca por novos fármacos fotossensibilizantes de segunda geração, com absorção de longo comprimento de onda, foi relatada por Wei Liu e colaboradores ${ }^{22}$. O objetivo do grupo era aplicar esses novos fármacos sintetizados na terapia fotodinâmica de tumores, dos quais foram estudadas novas ftalocianinas solúveis em água.

Christopher Farley e colaboradores ${ }^{23}$ publicaram em 2016, no qual apresentaram um estudo fundamental de uma série de derivados de $\mathrm{ZnF} 16 \mathrm{Pc}$ nos quais os átomos de flúor aromático são progressivamente substituídos por tioalcanos. Os resultados obtidos por eles estão correlacionados aos cálculos de DFT, que mostram que as energias orbitais e simetrias são fatores importantes para explicar a dinâmica do estado excitado.

Alexandra V. Soldatova e colaboradores ${ }^{24}$ publicaram em 2006 um artigo, no qual os autores relataram que ocorreu investigação por espectrometria de absorção transiente ultrarrápida e métodos de DFT/TDDFT de propriedades fotofísicas de um grupo de tetrapirróis concentrados em Ni (II). Após a verificação mais profunda foi constatado através da revisão sistemática que o trabalho possui nível baixo de relevância para desenvolvimento da pesquisa final. 


\section{Conclusões}

Realizar uma boa revisão sistemática é fundamental para o início de qualquer trabalho, pois é necessário buscar na literatura potenciais e fraquezas para o tema abordado e assim verificar a relevância e contribuição para o meio científico. Essa busca avançada mostrou que o tema proposto inicialmente deveria ser alterado, haja visto que outros autores já haviam publicado algo com mesma ideia abordada, no qual se baseava na explicação do processo fotoquímico ocorrido na vitamina $\mathrm{D}$. A organização dos dados através do programa StArt foi muito útil na tomada de decisão, após escolhas corretas das strings e inclusão e exclusão de artigos diversos. Dessa forma, optou-se pela estrutura de Tetraazaporfirina de Cobalto devido seu potencial, assim como outros derivados das porfirinas na aplicação em terapia fotodinâmica, atuando como transportadores de óxido nítrico, bastante conhecido por desempenhar papéis diversos em processos biológicos descritos na literatura, podendo destruir tecidos tumorais através de espécies tóxicas geradas com resultado da transferência de energia. Foram selecionados trabalhos diversos que estão relacionados com a linha de pesquisa proposta. Artigos que explicam estruturas derivadas das porfirinas através de rotinas práticas ou teóricas com níveis de teoria como DFT, e utilizando a mesma metodologia pretendida ao longo dessa pesquisa (dinâmica de estados excitados - Surface Hopping). O estudo teórico da Tetraazaporfirina de Cobalto relacionado a liberação de NO devido ao estímulo eletromagnético com frequência e comprimentos de ondas específicos contribui para a busca de possíveis aplicações farmacológicas dessa classe de compostos bem como para o meio científico.

\section{Referências}

1. Zhu, W. et al. An efficient tumor-inducible nanotheranostics for magnetic resonance imaging and enhanced photodynamic therapy. Chem. Eng. J. 358, 969-979 (2019).

2. Herold, S. Interaction of nitrogen monoxide with hemoglobin and the artefactual production of S-nitroso-hemoglobin. Comptes Rendus Biol. 326, 533-541 (2003).
3. Bartberger, M. D. et al. The reduction potential of nitric oxide (NO) and its importance to NO biochemistry. Proc. Natl. Acad. Sci. U. S. A. 99, 10958-10963 (2002).

4. Enemark, J. H. \& Feltham, R. D. Principles of structure, bonding, and reactivity for metal nitrosyl complexes. Coord. Chem. Rev. 13, 339-406 (1974).

5. Richter-Addo, G. B., Legzdins, P. \& Burstyn, J. Introduction: Nitric oxide chemistry. Chem. Rev. 102, 857-859 (2002).

6. Hunt, A. P. \& Lehnert, N. Heme-Nitrosyls: Electronic Structure Implications for Function in Biology. Acc. Chem. Res. 48, 21172125 (2015).

7. $\mathrm{Bi}, \mathrm{W}$. et al. A switchable NLO organic-inorganic compound based on conformationally chiral disulfide molecules and $\mathrm{Bi}(\mathrm{III}) \mathrm{I} 5$ iodobismuthate networks. Adv. Mater. 20, 1013-1017 (2008).

8. Aoki, K., Nagano, K. \& Iitaka, Y. The crystal structure of L -arginine phosphate monohydrate . Acta Crystallogr. Sect. B Struct. Crystallogr. Cryst. Chem. 27, 11-23 (1971).

9. Lima, J. M. M., Silva, V. H. C., Camargo, L. T. F. M., De Oliveira, H. C. B. \& Camargo, A. J. Theoretical investigation on ruthenium tetraazaporphyrin as potential nitric oxide carrier in biological systems. J. Mol. Model. 19, 1727-1737 (2013).

10. Worth, G. A. \& Cederbaum, L. S. Beyond born-oppenheimer: Molecular dynamics through a conical intersection. Annu. Rev. Phys. Chem. 55, 127-158 (2004).

11. Barbatti, M. Nonadiabatic dynamics with trajectory surface hopping method. Wiley Interdiscip. Rev. Comput. Mol. Sci. 1, 620-633 (2011).

12. Yonehara, T., Hanasaki, K. \& Takatsuka, K. Fundamental approaches to nonadiabaticity: Toward a chemical theory beyond the bornoppenheimer paradigm. Chem. Rev. 112, 499-542 (2012).

13. Nikitin EE. Der gegenwärtige Stand der Theorien bimolekularer Reaktionen. Berichte der Bunsengesellschaft für Phys Chemie. 1968; 72(8): 949-959.

14. Tully, J. C. \& Pkeston, R. K. Trajectory surface hopping approach to nonadiabatic molecular collisions: The reaction of $\mathrm{H}+$ with $\mathrm{D} 2$. J. Chem. Phys. 55, 562-572 (1971).

15. Curchod, B. F. E., Rothlisberger, U. \& Tavernelli, I. Trajectorybased nonadiabatic dynamics with time-dependent density functional theory. ChemPhysChem 14, 1314-1340 (2013).

16. Akimov, A. V., Neukirch, A. J. \& Prezhdo, O. V. Theoretical insights into photoinduced charge transfer and catalysis at oxide interfaces. Chem. Rev. 113, 4496-4565 (2013).

17. Cisneros, C., Thompson, T., Baluyot, N., Smith, A. C. \& Tapavicza, E. The role of tachysterol in Vitamin D photosynthesis-a nonadiabatic molecular dynamics study. Phys. Chem. Chem. Phys. 19, 5763-5777 (2017). 
18. Maiti, B. et al. Photoinduced Homolytic Bond Cleavage of the Central Si-C Bond in Porphyrin Macrocycles Is a Charge Polarization Driven Process. J. Phys. Chem. A 120, 7634-7640 (2016).

19. Dini, D., Hanack, M., Egelhaaf, H. J., Sancho-Garcia, J. C. \& Cornil, J. Synthesis of axially substituted tetrapyrazinoporphyrazinato metal complexes for optical limiting and study of their photophysical properties. J. Phys. Chem. B 109, 5425-5432 (2005).

20. Kim, P. et al. Ultrafast intramolecular energy relaxation dynamics of benzoporphyrins: Influence of fused benzo rings on singlet excited states. J. Phys. Chem. B 115, 3784-3792 (2011).

21. Xu, Z., Gao, F., Makarova, E. A., Heikal, A. A. \& Nemykin, V. N. Energy transfer from colloidal quantum dots to near-infraredabsorbing tetraazaporphyrins for enhanced light harvesting. J. Phys. Chem. C 119, 9754-9761 (2015).

22. Liu, W., Kumar, M. R., Vicente, M. G. H., Fronczek, F. R. \& Smith, K. M. New water-soluble phthalocyanines and other terapyrroles for application in photodynamic therapy. Opt. Methods Tumor Treat. Detect. Mech. Tech. Photodyn. Ther. XIV 5689, 39 (2005).

23. Farley, C., Bhupathiraju, N. V. S. D. K., John, B. K. \& Drain, C. M. Tuning the Structure and Photophysics of a Fluorous Phthalocyanine Platform. J. Phys. Chem. A 120, 7451-7464 (2016).

24. Soldatova, A. V. et al. Effects of benzoannulation and $\alpha$-octabutoxy substitution on the photophysical behavior of nickel phthalocyanines: A combined experimental and DFT/TDDFT study. Inorg. Chem. 46, 2080-2093 (2007).

\section{Igor C. Morais, João L. P. Lisboa, José A. S. Neto, Karolina S. Gonçalves*, Oliver A. J. S. Filho \& Rosemberg F. N. Rodrigues}

Centro Universitário de Anápolis - UniEVANGÉLICA. Avenida Universitária, km 3,5, Centro Universitário, Anápolis, Goiás, Brasil.

E-mail: karol.souza948@gmail.com 